\title{
Supervised slackline training improves postural stability
}

\author{
JÜRGEN PFUSTERSCHMIED ${ }^{1}$, MICHAEL BUCHECKER ${ }^{1}$, MARTIN KELLER $^{2}$, \\ HERBERT WAGNER $^{1}$, WOLFGANG TAUBE ${ }^{2}$, \& ERICH MÜLLER ${ }^{1}$ \\ ${ }^{1}$ Christian Doppler Laboratory 'Biomechanics in skiing', Department of Sport Science and Kinesiology, University of \\ Salzburg, Schlossallee 49, Hallein/Rif, 5400 Austria, and ${ }^{2}$ Unit of Sports Science, Department of Medicine, University of \\ Fribourg, Chemin du Musée 3, Fribourg, 1700 Switzerland
}

\begin{abstract}
The present study investigated whether or not four weeks of supervised slackline training (SLT) performed on nylon webbing improves postural stability. Twenty-four healthy adults participated in the study and were assigned to either SLT $(n=12)$ or a control $(\mathrm{CON})$ group $(n=12)$. The SLT group completed a four week training program, while the CON group received no training. Centre of gravity (COG) and joint angles (ankle, knee and hip) were calculated using whole body three-dimensional (3D) kinematic measurements during single leg standing on a stable surface (SS) and on a perturbed surface (PS) before and after training. After SLT, a significant interaction was found for the SS condition in anteriorposterior COG mean velocity, whereas no changes were observed in the medio-lateral direction or in joint kinematics. In the PS condition, the medio-lateral COG mean velocity, frontal angular range of motion in the knee and hip joint, sagittal angular mean velocity in the knee joint, as well as frontal angular mean velocity in the hip joint were reduced in the SLT group only (all $P<0.05$ ). No significant training effects were detected in the ankle joint kinematics in either group from pre to post test. Our findings demonstrate that four weeks of supervised SLT improves postural stability in single leg stance on a stable surface and/or during compensation of perturbations.
\end{abstract}

Keywords: Training, balance, 3D kinematics, stable and perturbed surfaces

\section{Introduction}

In recent years a new upcoming recreational sport known as 'slacklining', has become popular with children and young adults (Balcom, 2005; Kleindl, 2010; Miller \& Friesinger, 2009). A slackline is composed of nylon webbing and is stretched tight enough between two anchor points that people can balance on it (Balcom, 2005). Depending on the length and the tension of the slackline, the line characteristics (amplitude and frequency of the line sway) can be altered and thus, the task difficulty can be adjusted. In general, the line has highly elastic properties and is in this respect similar to a trampoline. However, it offers only a small non-fixed base of support (Figure 1). This highly movable base of support may also be considered as the main differ- ence between slacklining and 'classical balance training'. In classical balance training, all devices are more or less unvarying in their position in space (Taube, Leukel, \& Gollhofer, 2008).

Several neural systems, different movement strategies and musculoskeletal components such as range of motion (ROM) of joints are involved in order to maintain the body's position against gravity (Shumway-Cook \& Woollacott, 2007). Based on the high postural demands associated with balancing on a slackline it might be assumed that slackline training (SLT) may not only improve the ability to balance on the slackline but also improve postural control in other balance situations (i.e. skiing or skating). Therefore, the results of Granacher and coworkers (Granacher, Iten, Roth, \& Gollhofer, 2010) were rather surprising as their participants did not de- 


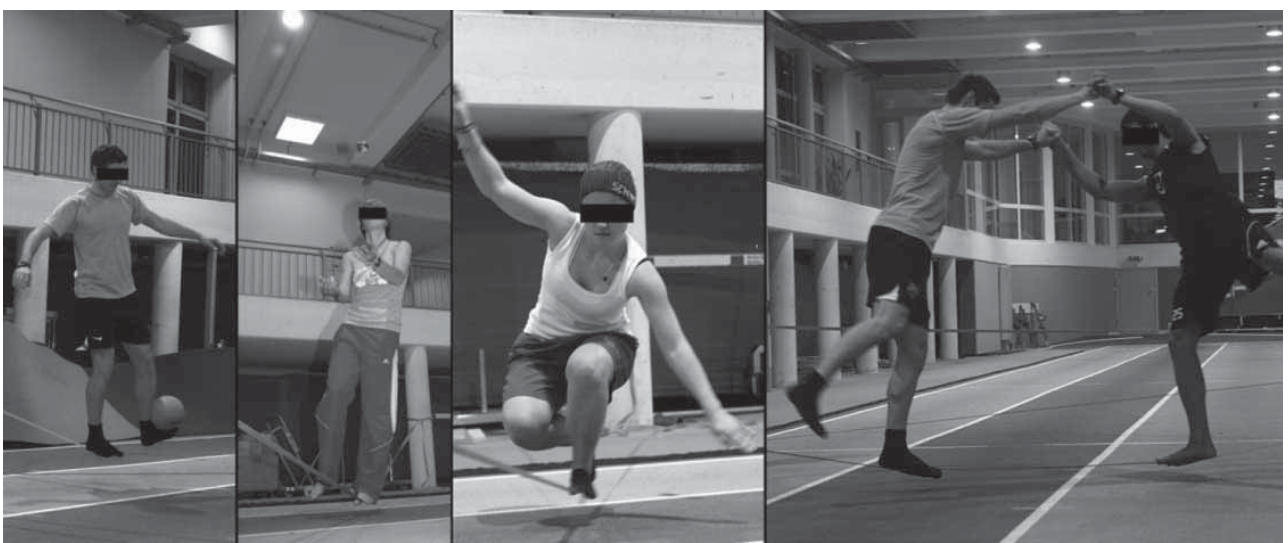

Figure 1. Balancing on the slacklines and performing additional tasks in the fourth week of training.

monstrate any improvements in postural control after four weeks of SLT when assessed during postural tasks other than slacklining. Based on previous classical balance training studies showing transferability of postural skills to untrained situations (Beck et al., 2007; Granacher, Gollhofer, \& Strass, 2006; Taube et al., 2007) it was hypothesised that adaptations after SLT may not be restricted to the task itself but may help to improve balance performance in a more general way. Thus, the purpose of this study was to determine the effects of four weeks of SLT on single leg balance performance on a stable and a perturbed surface by means of whole body three-dimensional (3D) kinematic measures in healthy young adults.

\section{Materials and methods}

\section{Participants}

A sample of 24 healthy young adults (Table I) without a history of musculoskeletal disorders was recruited for the study. None of the volunteers had experience with systematic balance or SLT. After the experiment was explained, each participant signed a

Table I. Characterisics of the participants taking part in the study $($ mean \pm SE)

\begin{tabular}{lcc}
\hline Demographics & CON $(n=12)$ & SLT $(n=12)$ \\
\hline body height $(\mathrm{cm})$ & $167.3 \pm 2.1$ & $174.1 \pm 2.6$ \\
body mass $(\mathrm{kg})$ & $62.5 \pm 2.7$ & $68.3 \pm 3.2$ \\
age (years) & $25.7 \pm 0.8$ & $23.3 \pm 1.0$ \\
activity level (h/week) & $6.2 \pm 1.7$ & $5.4 \pm 0.7$ \\
sex (female/male) & $6 / 6$ & $5 / 7$ \\
\hline
\end{tabular}

During 4-weeks activity level (every day activity like biking, walking, running, etc.) was assessed by questionnaire. The training intervention was disregarded in the table and statistical analysis. No statistical significant differences $(P>0.05)$ between CON and SLT groups were found concerning their body height; body mass, age and activity level. document of informed consent that was approved by the local ethics commission before participating in the study.

\section{Slackline training}

Participants were randomly assigned to either the experimental SLT group or to the Control (CON) group. The SLT group performed 10 balance training sessions using a slackline for four weeks on nonconsecutive days (Table II), while the CON group received no training. All SLT sessions were held in an athletic-hall with pillars used as anchors to fix the nylon webbings about $50-70 \mathrm{~cm}$ above the floor. The same type of slackline (width: $25.8 \mathrm{~mm}$; diameter: $2.49 \mathrm{~mm}$; fracture strain: $4.8 \%$ ) was used in different lengths $(7-18 \mathrm{~m})$. All training sessions lasted $90 \mathrm{~min}$. Participants started with a warm up, followed by training on the slacklines, and ended with a short cool down exercise. The methodological contents of SLT were based on the literature (Balcom, 2005; Kleindl, 2010; Miller \& Friesinger, 2009) and on personal experience of the authors gained in previously conducted slackline workshops. In the first week of training, participants learned to step up and to stand

Table II. Overview of the slackline training protocol

\begin{tabular}{llc}
\hline Week & \multicolumn{1}{c}{ Exercises and content of teaching } & $\begin{array}{c}\text { Length of the } \\
\text { line }(\mathrm{m})\end{array}$ \\
\hline $1 \quad$ Standing and first steps with support & $7-10$ \\
& Standing and first steps with minor support & $7-10$ \\
& Standing and steps without support & $7-10$ \\
2 & Walking forward and backward & $10-12$ \\
& Catch and pass a ball & $10-12$ \\
3 & Turns on the line & $10-12$ \\
& Standing up from a sitting position & $12-18$ \\
4 & Juggling balls & $12-18$ \\
& Walking with constraints (e.g. arms akimbo) & $12-18$ \\
& Two people standing on one line & $12-18$ \\
\hline
\end{tabular}


on short slacklines (i.e., 7-10 m). After the second week of training, all participants were able to walk a few steps on the short line without assistance. During the four weeks, task-difficulty was constantly increased by lengthening the slacklines, changing the line tension and performing additional tasks (Figure 1) during balancing (e.g. closing eyes, walking backwards, juggling with balls).

\section{Testing procedure}

Posturographic measurements were executed during a $15 \mathrm{~s}$ single leg stance on a stable surface (SS) and on a perturbed surface (PS) before (PRE) and after (POST) the SLT intervention. The medio-lateral perturbations were performed on a multi-axial free swinging platform (Posturomed, Haider Bioswing, Pullenreuth, Germany) (Boeer, Mueller, Krauss, Haupt, \& Horstmann, 2010; Muller, Gunther, Krauss, \& Horstmann, 2004). The platform was deflected in a medio-lateral direction and magnetically fixed with a maximum displacement of approximately $25 \mathrm{~mm}$. After detaching the magnet, a highly reliable perturbation stimulus could be provoked, which was indicated by an average peak net cross correlation $\left(R_{\mathrm{xy}}^{2}\right)$ of 0.97 over 10 trials. When participants were in a controlled testing-position, the perturbation impulse was applied at random times. Participants were instructed to stand barefoot as motionless as possible while bending their stance knee slightly and looking at a stationary target placed $5 \mathrm{~m}$ in front of them. They were encouraged to minimise their postural sway in the SS condition and to regain equilibrium as quickly as possible after the perturbation. To familiarise the participants with each situation and verify their understanding of instructions, preliminary practice trials were given (Benvenuti et al., 1999; Riemann, Myers, \&
Lephart, 2003). In the SS condition, the participants positioned their hands on their iliac crests. During the PS condition the hands and arms were stretched sideward. Two trials in the SS condition and three trials in the PS condition were recorded and averaged. Between consecutive trials, a rest interval of $20 \mathrm{~s}$ was given. A trial was defined as invalid and was repeated, if the participant touched the ground with their contra-lateral leg or grasped the handrails of the Posturomed.

\section{Instrumentation and data analysis}

A passive, 3D motion analysis system (VICON MX13, Vicon, Oxford, UK), including eight cameras sampling at $250 \mathrm{~Hz}$, was used to collect kinematic data. The accuracy of the recording system, verified by calculating the root mean square errors for each camera, ranged from 0.15 to $0.26 \mathrm{~mm}$ during calibration. Thirty-nine reflective markers (14 $\mathrm{mm}$ in diameter) were attached to anatomic landmarks in order to estimate joint centres, segment orientation, whole-body centre of gravity (COG) location and joint angles using commercially available software (Nexus, Vicon, Oxford, UK) and the PlugIn Gait model (Vicon, Oxford, UK). As shown in Table III, further analysis focused on elementary variables computed from COG trajectories in anterior-posterior (AP) or ' $y$ ' direction and medio-lateral (ML) or ' $x$ ' direction, as well as from ankle, knee and hip joint motion in the sagittal and frontal plane. In the SS condition, the first $5 \mathrm{~s}$ were excluded from analysis to minimise interference from initial preparation, and in the PS condition data were analysed beginning from the onset of perturbation, so that in both conditions $10 \mathrm{~s}$ were used to calculate the variables of interest (Benvenuti et al., 1999; Riemann et al., 2003). All

Table III. List of abbreviations and description of the kinematic variables

\begin{tabular}{ll}
\hline Abbreviation & \multicolumn{1}{c}{ Variable Definition $^{\text {a }}$} \\
\hline COG-PEx & peak-to-peak excursion of the center of gravity in medio-lateral direction \\
pOG-PEy & meak-to-peak excursion of the center of gravity in anterior-posterior direction \\
COG-MVx & mean velocity of COG/COG-H in AP direction \\
COG-MVy & peak-to-peak excursion of the plantar-/dorsiflextion ankle angle \\
AA-PEx & peak-to-peak excursion of the e-/inversion ankle angle \\
AA-PEy & mean velocity displacement of the e-/inversion ankle angle \\
AA-MVx & mean velocity displacement of the plantar-/dorsiflextion ankle angle \\
AA-MVy & peak-to-peak excursion of the flex-/extension knee angle \\
KA-PEx & peak-to-peak excursion of the ad-/abduction knee angle \\
KA-PEy & mean velocity displacement of the flex-/extension knee angle \\
KA-MVx & mean velocity displacement of the ad-/abduction knee angle \\
KA-MVy & peak-to-peak excursion of the flex-/extension hip angle \\
HA-PEx & peak-to-peak excursion of the ad-/abduction hip angle \\
HA-PEy & mean velocity displacement of the flex-/extension hip angle \\
HA-MVx & mean velocity displacement of the ad-/abduction hip angle \\
HA-MVy & $\mathrm{m}^{-1} \cdot \mathrm{s}^{-1} \cdot \mathrm{m}^{-1}$ \\
\hline
\end{tabular}

${ }^{\mathrm{a}} \mathrm{COG}$ (centre of gravity); COG-H (height of COG); ML (medio-lateral); AP (anterior-posterior). 
trajectories were transferred to a custom written computer program (MatLab, The MathWorks Inc., Natick, USA) to conduct post-processing. Data analysis included smoothing (at $6 \mathrm{~Hz}$ using a fourth order, phase-corrected, low-pass Butterworth filter) (Benvenuti et al., 1999), calculation of variables and statistical analysis.

\section{Statistical analysis}

A MANOVA was used to determine possible PRE test differences between the SLT and CON groups. All kinematic variables were analysed for the SS and PS conditions. Separate $2 \times 2$ [time (PRE and POST $) \times$ group $($ SLT and CON)] mixed-design repeated measures ANOVAs were used to test for interaction effects, global differences in the dependent variables between PRE and POST and possible differences between SLT and CON. Additionally, paired samples (two-tailed) t-tests were applied to identify changes within each group from PRE to POST test. For all statistical analyses, the level of significance was set to $P<0.05$. Additionally, effect sizes $(f)$, were calculating and reported using convention by Cohen: $f=0.10$ indicates small, $f=0.25$ medium, and $f=0.40$ large effects (Aron, Aron, \& Coups, 2006; Cohen, 1988).

\section{Results}

For SS and PS, means, standard errors (SE) and effect sizes $(f)$ for main effects (time, group) and group $\times$ time interactions for all dependent variables are listed in Tables IV and V. No differences in dependent variables were found between the SLT and CON groups at PRE test. Concerning the SS condition, a significant training effect $(P<0.05$, $f=0.49$ ), as well as a significant main effect of group $(P<0.05, f=0.55)$ was observed in COG-mean velocity in the y direction (MVy). Post hoc comparisons showed a significant decrease in sway scores by the SLT group $(P<0.01)$, whereas no changes $(P>0.05)$ were observed in the CON group. In addition, a main effect for group in the variables hip angle (HA)-MVx $(P<0.05, f=0.47)$ and HA-MVy $(P<0.05, f=0.45)$ was revealed. For all other measured variables, no group $\times$ time interactions and no main effects of group or time (all $P>0.05$ ) were observed. In the PS condition, significant group $\times$ time interactions for the variables COG$\operatorname{MVx}(P<0.05, f=0.46)$, knee angle-peak excursion $($ KA-PE)y $(P<0.05, f=0.46)$, KA-MVx $(P<0.05, f$ $=0.54)$, HA-PEy $(P<0.01, f=0.82)$, and HAMVy $(P<0.05, f=0.58)$ were revealed. For the SLT group, PRE to POST test scores improved by decreasing $(P<0.01)$ in all variables with a significant group $\times$ time interaction. The CON group did show a decrease in POST test scores for KA-MVx $(P<0.05)$ and HA-MVy $(P<0.05)$, whereas no significant changes were found for the variables COG-MVy, KA-PEy and HA-PEy (all $P>0.05$ ). Furthermore, main effects of time were significant $(P<0.05)$ in all variables except COG-PEx and

Table IV. Postural control variables in single leg standing on stable surface by group (mean \pm SE) and effect sizes $(f)$ for time, group and interaction effects

\begin{tabular}{|c|c|c|c|c|c|c|c|}
\hline \multirow[b]{2}{*}{ Variables $^{\mathrm{a}}$} & \multicolumn{2}{|c|}{ PRE } & \multicolumn{2}{|c|}{ POST } & \multicolumn{3}{|c|}{ Effect sizes $(f)$} \\
\hline & SLT & $\mathrm{CON}$ & SLT & $\mathrm{CON}$ & TIME & GROUP & $\mathrm{TxG}$ \\
\hline COG-PEx & $15.06 \pm 1.30$ & $14.79 \pm 1.16$ & $13.79 \pm 0.75$ & $14.56 \pm 1.22$ & 0.15 & 0.05 & 0.10 \\
\hline COG-PEy & $18.67 \pm 1.20$ & $18.62 \pm 1.97$ & $15.48 \pm 0.73^{\$}$ & $19.56 \pm 1.41$ & 0.23 & 0.25 & 0.42 \\
\hline COG-MVx & $4.90 \pm 0.32$ & $5.08 \pm 0.35$ & $4.47 \pm 0.16$ & $5.00 \pm 0.27$ & 0.25 & 0.22 & 0.18 \\
\hline COG-MVy & $5.07 \pm 0.23$ & $5.45 \pm 0.31$ & $4.28 \pm 0.26^{\$ \$}$ & $5.75 \pm 0.39$ & 0.23 & $0.55^{\star}$ & $0.49^{\star}$ \\
\hline AA-PEx & $2.24 \pm 0.27$ & $2.55 \pm 0.25$ & $2.41 \pm 0.26$ & $2.37 \pm 0.29$ & 0.00 & 0.09 & 0.18 \\
\hline AA-PEy & $1.64 \pm 0.24$ & $1.82 \pm 0.20$ & $1.56 \pm 0.23$ & $1.70 \pm 0.21$ & 0.11 & 0.14 & 0.02 \\
\hline AA-MVx & $1.59 \pm 0.17$ & $1.65 \pm 0.13$ & $1.63 \pm 0.18$ & $1.71 \pm 0.23$ & 0.07 & 0.07 & 0.02 \\
\hline AA-MVy & $1.37 \pm 0.17$ & $1.60 \pm 0.20$ & $1.37 \pm 0.22$ & $1.50 \pm 0.23$ & 0.08 & 0.15 & 0.08 \\
\hline KA-PEx & $2.90 \pm 0.46$ & $2.66 \pm 0.30$ & $2.53 \pm 0.17$ & $2.64 \pm 0.33$ & 0.14 & 0.04 & 0.13 \\
\hline KA-PEy & $0.97 \pm 0.16$ & $1.14 \pm 0.09$ & $0.92 \pm 0.10$ & $1.51 \pm 0.36$ & 0.18 & 0.36 & 0.23 \\
\hline KA-MVx & $1.81 \pm 0.17$ & $2.14 \pm 0.27$ & $1.57 \pm 0.17$ & $2.18 \pm 0.33$ & 0.11 & 0.33 & 0.16 \\
\hline KA-MVy & $0.85 \pm 0.11$ & $0.99 \pm 0.10$ & $0.82 \pm 0.09$ & $1.42 \pm 0.35$ & 0.24 & 0.36 & 0.28 \\
\hline HA-PEx & $1.99 \pm 0.22$ & $2.33 \pm 0.27$ & $2.02 \pm 0.26$ & $2.44 \pm 0.29$ & 0.06 & 0.28 & 0.04 \\
\hline HA-PEy & $1.87 \pm 0.24$ & $2.16 \pm 0.29$ & $1.99 \pm 0.26$ & $2.04 \pm 0.34$ & 0.00 & 0.12 & 0.09 \\
\hline HA-MVx & $1.10 \pm 0.08$ & $1.39 \pm 0.14$ & $1.02 \pm 0.09$ & $1.42 \pm 0.19$ & 0.04 & $0.47^{\star}$ & 0.10 \\
\hline HA-MVy & $0.91 \pm 0.09$ & $1.29 \pm 0.15$ & $0.84 \pm 0.05$ & $1.19 \pm 0.25$ & 0.13 & $0.45^{\star}$ & 0.02 \\
\hline
\end{tabular}

There were no significant differences found between INT group and CON group in pre test. In all significant TxG interactions, there was a significant decrease in scores at the post-test for the INT group compared with the pre test values.

$\$(P<0.05), \$(P<0.01)$ indicates significant differences form pre to post test.

${ }^{\star}(P<0.05),{ }^{\star \star}(P<0.01)$ indicates significant main effects and interactions.

${ }^{a}$ Description and units of variables are listed in Table II. 
Table V. Postural control variables in single leg standing on perturbed surface by group (mean \pm SE) and effect sizes $(f)$ for time, group and interaction effects

\begin{tabular}{|c|c|c|c|c|c|c|c|}
\hline \multirow[b]{2}{*}{ Variables $^{\mathrm{a}}$} & \multicolumn{2}{|c|}{ PRE } & \multicolumn{2}{|c|}{ POST } & \multicolumn{3}{|c|}{ Effect sizes $(f)$} \\
\hline & SLT & $\mathrm{CON}$ & SLT & $\mathrm{CON}$ & TIME & GROUP & TxG \\
\hline COG-PEx & $39.59 \pm 3.77$ & $35.90 \pm 2.59$ & $36.30 \pm 3.16$ & $33.12 \pm 1.92$ & 0.28 & 0.21 & 0.02 \\
\hline COG-PEy & $28.54 \pm 2.19$ & $26.68 \pm 1.84$ & $26.11 \pm 1.84$ & $25.56 \pm 1.39$ & 0.24 & 0.13 & 0.09 \\
\hline COG-MVx & $15.22 \pm 1.88$ & $11.58 \pm 1.16$ & $9.82 \pm 0.85^{\$ \$}$ & $10.09 \pm 0.81$ & $0.80^{\star \star}$ & 0.24 & $0.46^{\star}$ \\
\hline COG-MVy & $9.78 \pm 0.52$ & $9.36 \pm 0.67$ & $7.80 \pm 0.47^{\$ \$}$ & $8.29 \pm 0.43^{\$}$ & $1.01^{\star \star}$ & 0.01 & 0.30 \\
\hline AA-PEx & $6.61 \pm 0.64$ & $5.84 \pm 0.54$ & $5.47 \pm 0.64$ & $5.00 \pm 0.41$ & $0.49^{\star}$ & 0.20 & 0.07 \\
\hline AA-PEy & $4.80 \pm 0.81$ & $5.36 \pm 0.49$ & $4.26 \pm 0.62$ & $4.42 \pm 0.39$ & $0.51^{\star}$ & 0.10 & 0.14 \\
\hline AA-MVx & $5.06 \pm 0.62$ & $3.98 \pm 0.34$ & $3.18 \pm 0.32^{\$ \$}$ & $3.26 \pm 0.29^{\$}$ & $0.84^{\star \star}$ & 0.22 & 0.37 \\
\hline AA-MVy & $4.54 \pm 0.83$ & $4.07 \pm 0.62$ & $2.78 \pm 0.41^{\$}$ & $2.91 \pm 0.28^{\$}$ & $0.82^{\star \star}$ & 0.05 & 0.17 \\
\hline KA-PEx & $10.89 \pm 0.91$ & $9.98 \pm 1.04$ & $8.19 \pm 0.82^{\$ \$}$ & $8.55 \pm 0.77$ & $0.82^{\star \star}$ & 0.05 & 0.25 \\
\hline KA-PEy & $5.53 \pm 0.64$ & $4.74 \pm 0.40$ & $3.65 \pm 0.37^{\$ \$}$ & $4.33 \pm 0.44$ & $0.72^{\star \star}$ & 0.02 & $0.46^{\star}$ \\
\hline KA-MVx & $6.74 \pm 0.68$ & $5.24 \pm 0.42$ & $3.94 \pm 0.25^{\$ \$}$ & $4.28 \pm 0.34^{\$}$ & $1.11^{\star \star}$ & 0.23 & $0.54^{\star}$ \\
\hline KA-MVy & $4.75 \pm 0.60$ & $4.05 \pm 0.64$ & $2.85 \pm 0.30^{\$ \$}$ & $3.13 \pm 0.45$ & $0.77^{\star \star}$ & 0.07 & 0.27 \\
\hline HA-PEx & $9.42 \pm 0.83$ & $8.64 \pm 0.92$ & $6.65 \pm 0.77^{\$ \$}$ & $7.37 \pm 0.52$ & $0.92^{\star \star}$ & 0.01 & 0.34 \\
\hline HA-PEy & $14.06 \pm 0.97$ & $14.77 \pm 1.72$ & $9.65 \pm 0.58^{\$ \$}$ & $14.33 \pm 1.82$ & $1.00^{\star \star}$ & 0.31 & $0.82^{\star \star}$ \\
\hline HA-MVx & $4.70 \pm 0.52$ & $4.15 \pm 0.41$ & $2.96 \pm 0.20^{\$ \$}$ & $3.02 \pm 0.21^{\$ \$}$ & $1.05^{\star \star}$ & 0.12 & 0.22 \\
\hline HA-MVy & $7.34 \pm 1.00$ & $5.78 \pm 0.78$ & $3.74 \pm 0.30^{\$ \$}$ & $4.79 \pm 0.52^{\$}$ & $1.03^{\star \star}$ & 0.06 & $0.58^{\star}$ \\
\hline
\end{tabular}

There were no significant differences found between INT group and CON group in pre test. In all significant TxG interactions, there was a significant decrease in scores at the post test for the INT group compared with the pre test values.

${ }^{\$}(P<0.05),{ }^{\$ \$}(P<0.01)$ indicates significant differences form pre to post test.

$\star(P<0.05),{ }^{\star \star}(P<0.01)$ indicates significant main effects and interactions.

${ }^{a}$ Description and units of variables are listed in Table II.

COG-PEy (both $P>0.05$ ). No main effects of group were detected $(P>0.05)$.

\section{Discussion}

The main finding of this study is that SLT improves postural control during stable and perturbed single leg stance (Figure 2), which is provided by large effect sizes in both conditions. These results confirm our initial hypothesis that balance skills acquired from SLT can be transferred to other postural tasks. The present results appear to be broadly in accordance with the literature, regarding the effects of classical balance and coordination training on postural stability (Emery, Cassidy, Klassen, Rosychuk, \& Rowe, 2005; Paterno, Myer, Ford, \& Hewett, 2004; Rasool \& George, 2007). Although Granacher et al. (Granacher, Iten, Roth, \& Gollhofer, 2010) were not able to show improvements in balance, this result is not self-evident as other studies have demonstrated a strong task specificity for trainingrelated balance adaptations (Beck et al., 2007; Taube et al., 2008). Based on the altered Range of Motion (ROM) in knee and hip joints after SLT, it may be speculated that this adaptation is beneficial for improving joint stability which has been shown to reduce risk of lower limb injury (Hewett et al., 2005; Myer, Ford, McLean, \& Hewett, 2006). Thus, SLT may have similar preventive characteristics as classical balance training (DiStefano, Clark, \& Padua, 2009; Emery et al., 2005; Hubscher et al., 2010; Hupperets, Verhagen, \& van Mechelen, 2009;
McGuine \& Keene, 2006; Myklebust et al., 2003; Valovich McLeod, 2008).

\section{Slackline training improves postural control}

Regarding the SS condition, four weeks of SLT significantly reduced AP COG sway; but, it did not change ML body sway nor did it affect lower limb kinematics similarly. The SLT participants were able to reduce their COG sway in the AP direction using a variety of strategies. Some participants reduced peak excursion of their ankle deflection $(n=4)$ and others reduced movements of the knee $(n=6)$ or hip $(n=7)$. This seems surprising as it is widely accepted that during quiet stance on stable surfaces, the body is primarily controlled by subtle ankle deflections with minimal involvement of hip or knee joints (Amiridis, Arabatzi, Violaris, Stavropoulos, \& Hatzitaki, 2005; Shumway-Cook \& Woollacott, 2007; Williams, Chmielewski, Rudolph, Buchanan, \& Snyder-Mackler, 2001). Thus, one could argue that ankle kinematics should have changed to explain the reduction in COG displacement in the POST measurement. However, when considering the training task of slacklining one can appreciate the need by the participants to compensate for much larger deflections of the line which would require considerably more movement of the hips and knees. It is therefore conceivable that slacklining can effectively enhance motor control in knee and hip joints (Myer et al., 2006), a training 

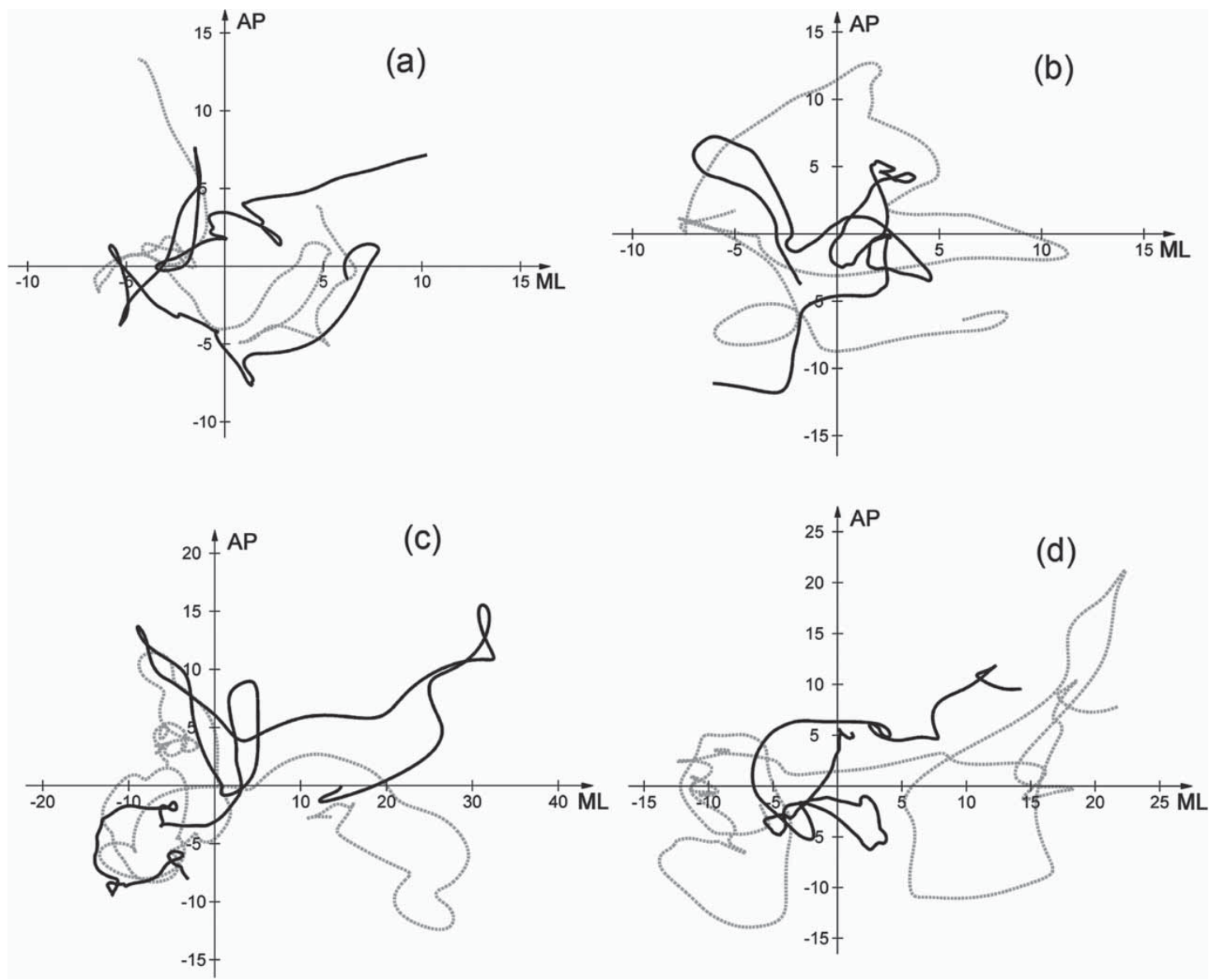

Figure 2. Representative centre of gravity (COG, $\mathrm{mm}$ ) data in anterior-posterior (AP) and medio-lateral (ML) direction during stable (a,b) and perturbed $(c, d)$ stance. Plots on the left side $(a, c)$ show COG movement of a participant in the control group, where the plots on the right side $(b, d)$ demonstrate the COG sway of a participant of the slackline training group. Gray dashed line shows the COG sway in pretesting and solid black line indicates the COG sway in post-testing. The largest changes in COG sway were observed in the slackline training group during perturbed stance (d).

effect clearly shown by our data during perturbed stance.

In contrast to the SS condition, the number of moderate and large effects was much larger than in the PS condition. During the compensation of perturbations, the ML COG sway, sagittal angular velocity at the knee, as well as frontal angular ROM at the knee and hip joint were significantly reduced after SLT. There are several possible mechanisms that could account for the improved balance performance during perturbations. First, large perturbations have been shown to be compensated for by corrective motions at the hip joint (Amiridis et al., 2005; Riemann et al., 2003; Shumway-Cook \& Woollacott, 2007). This finding was also confirmed in our study. The largest frontal angular movements during perturbation were observed at the hip. Interestingly, the decreased ML COG sway, presumably as a result of training, was accompanied by a similar decreased frontal angular ROM at the knee and hip. Consequently, it can be assumed that the reduction of ML COG sway could be related to the decreased frontal angular ROM at the knee and hip. Although we did not measure muscle activity, the reduced ROM at the knee and hip joint may be a result of increased joint stiffness as a consequence of enhanced activity of muscles encompassing the joint (Gruber, Bruhn, \& Gollhofer, 2006; Pedersen, Dye, Bergenheim, \& Djupsjöbacka, 2000; Rietdyk, Patla, Winter, Ishac, \& Little, 1999; Williams et al., 2001). In summary fewer improvements were found in the SS than in the PS condition. However, this is explainable due to the fact, that slacklining is a high dynamic movement and therefore the need for regaining equilibrium is much greater than the need for maintaining balance.

The finding of improved balance control in our study is contrary to the results of Granacher and coworkers (Granacher et al., 2010), who found no change in balancing skill after four weeks of SLT. Possible explanations for this discrepancy may be that in the present study, the COG sway was assessed 
whereas Granacher et al. (2010) computed total centre of pressure (COP) displacement. Different methods of data acquisition and data analysis might have led to inconsistent findings. A comparison between $\mathrm{COP}$ and $\mathrm{COG}$ measures regarding longterm reliability would favour COG values (Benvenuti et al., 1999). Therefore, the methodology used in this study may be more appropriate for balance studies versus more commonly used force plate measurements such as the one used by Granacher et al. (2010). A second possible explanation is of a more technical origin: In the present study the width of the slackline was smaller and its length greater compared to Granacher et al. (2010). Thus, the demands to balance on the slackline were probably higher. Perhaps a certain degree of task complexity is necessary to provoke improvements in balance performance. Additionally, the training sessions differed in time between the two studies. Whereas participants in the present study trained for $90 \mathrm{~min}$ per session participants in the study of Granacher et al. (2010) only trained for $60 \mathrm{~min}$ per session. Therefore they only trained for two-thirds of the total time that participants in the present study trained. It is therefore possible that the total training time needs to be examined to determine if there is a minimal amount of time required to demonstrate an adaptive change to balancing ability. In summary, the quality of training stimuli as well as the overall duration of training was greater in the present study compared to the study by Granacher et al. (2010).

\section{Functional considerations}

Slackline training seems to be efficient to improve postural control and to reduce the ROM in the lower limb joints especially during perturbed single leg stance. Although balance improvements were also found during stable stance, but this minor challenging condition seems to be less suitable to detect changes in the joint ROM. Furthermore, Granacher et al. (2010) demonstrated that SLT can improve the maximal rate of force development. Therefore, at least some effects on balance and force skills after SLT seem to be comparable to the training effects reported after classical balance training (Taube et al., 2008). The fact that SLT significantly reduced the ROM of the knee and hip joint suggests it is possible that SLT and classical balance training have another characteristic in common; the prevention of lower limb injuries. Several studies have reported classical balance training and coordination training were associated with reduced incidence of lower limb injuries (DiStefano et al., 2009; Hubscher et al., 2010; McGuine, Greene, Best, \& Leverson, 2000; Wang, Chen, Shiang, Jan, \& Lin, 2006). It is speculated that balance training counteracts deficits in proprioception (joint sense), postural control and muscle imbalance and/or muscle weakness, which are considered intrinsic risk factors for sports injuries. Our findings indicate improvements in postural stability and a decreased frontal angular ROM at the knee and hip joint; but, no changes were observed in ankle joint kinematics. Thus, it may be reasonable to speculate that SLT may preferentially be suited to improve balance control thereby reducing the incidence of lower limb injuries. However, prospective studies have to clarify this assumption (Gruber et al., 2006).

\section{Limitations}

To the best of our knowledge, this is the first study to investigate the impact of supervised SLT on postural stability measured by 3D kinematics. The methodology used in this investigation was to a large extent comparable to previous studies investigating postural stability (Amiridis et al., 2005; Benvenuti et al., 1999; Madigan, Davidson, \& Nussbaum, 2006; Riemann et al., 2003) and indicated improved balance control as a result of SLT. Nevertheless, this study has some limitations. First, the population investigated in this study contains a relatively small sample size of young adults in good physical condition. It can be assumed that the impact of SLT would be different in untrained individuals than in a population of well-trained adults. Second, statistical analysis detected a significant interaction of group $\times$ time in the COG-MVy parameter when measured in the stable condition. However, this group $\times$ time effect became significant not only due to the improvements of the training group but also due to a slight (insignificant) deterioration of the CON group from PRE to POST test. Additionally, main effects of time for almost all variables were observed in the PS condition. This indicates a minor acquisition effect, which was also reported elsewhere (Boeer et al., 2010). This acquisition effect could however be counteracted by comparing the SLT group with the CON group. Thus, any differences between these groups are considered to indicate SLT-related adaptations. Another limitation of this study was the application of the perturbations only in the ML direction. This decision was based on the fact that, during slacklining, the line and the body moves primarily in the ML direction (Balcom, 2005; Kleindl, 2010; Miller \& Friesinger, 2009). Nevertheless, it would have been interesting to know whether and how the system adapts when confronted with perturbations in an AP direction. 


\section{Conclusion}

In summary, our study shows that four weeks of supervised SLT improves medio-lateral balance performance primarily during perturbed single leg stance. For this reason, we recommend slacklining as a suitable and inexpensive training exercise to improve regaining equilibrium in an upright position after perturbations. Further research should focus on the hip and knee joint encompassing muscles and should investigate muscle co-activation and reflex activity.

\section{References}

Amiridis, I., Arabatzi, F., Violaris, P., Stavropoulos, E., \& Hatzitaki, V. (2005). Static balance improvement in elderly after dorsiflexors electrostimulation training. European fournal of Applied Physiology, 94(4), 424-433.

Aron, A., Aron, E. N., \& Coups, E. J. (2006). Statistics for psychology. New Jersey: Pearson Prentice Hall.

Balcom, S. (2005). Walk the line. Ashland: SlackDaddy Press.

Beck, S., Taube, W., Gruber, M., Amtage, F., Gollhofer, A., \& Schubert, M. (2007). Task-specific changes in motor evoked potentials of lower limb muscles after different training interventions. Brain Research, 1179, 51-60.

Benvenuti, F., Mecacci, R., Gineprari, I., Bandinelli, S., Benvenuti, E., Ferrucci, L., et al. (1999). Kinematic characteristics of standing disequilibrium: Reliability and validity of a posturographic protocol. Archives of Physical Medicine and Rehabilitation, 80(3), 278-287.

Boeer, J., Mueller, O., Krauss, I., Haupt, G., \& Horstmann, T. (2010). Reliability of a measurement technique to characterise standing properties and to quantify balance capabilities of healthy subjects on an unstable oscillatory platform (Posturomed). Sportverletz Sportschaden, 24(1), 40-45.

Cohen, J. (1988). Statistical power for the behavioral sciences (2nd ed). Hillsdale, NJ: Lawrence Erlbaum Associates.

DiStefano, L. J., Clark, M. A., \& Padua, D. A. (2009). Evidence supporting balance training in healthy individuals: A systemic review. Fournal of Strength and Conditioning Research, 23(9), 2718-2731.

Emery, C. A., Cassidy, J. D., Klassen, T. P., Rosychuk, R. J., \& Rowe, B.H. (2005). Effectiveness of a home-based balancetraining program in reducing sports-related injuries among healthy adolescents: A cluster randomized controlled trial. Canadian Medical Association fournal, 172(6), 749-754.

Granacher, U., Gollhofer, A., \& Strass, D. (2006). Training induced adaptations in characteristics of postural reflexes in elderly men. Gait Posture, 24(4), 459-466.

Granacher, U., Iten, N., Roth, R., \& Gollhofer, A. (2010). Slackline training for balance and strength promotion. International Fournal of Sports Medicine, 31(10), 717-723.

Gruber, M., Bruhn, S., \& Gollhofer, A. (2006). Specific adaptations of neuromuscular control and knee joint stiffness following sensorimotor training. International fournal of Sports Medicine, 27(8), 636-641.

Hewett, T. E., Myer, G. D., Ford, K. R., Heidt, R. S., Jr., Colosimo, A. J., McLean, S. G., et al. (2005). Biomechanical measures of neuromuscular control and valgus loading of the knee predict anterior cruciate ligament injury risk in female athletes: A prospective study. American fournal of Sports Medicine, 33(4), 492-501.

Hubscher, M., Zech, A., Pfeifer, K., Hansel, F., Vogt, L., \& Banzer, W. (2010). Neuromuscular training for sports injury prevention: A systematic review. Medicine and Science in Sports and Exercise, 42(3), 413-421.

Hupperets, M. D., Verhagen, E. A., \& van Mechelen, W. (2009). Effect of unsupervised home based proprioceptive training on recurrences of ankle sprain: Randomised controlled trial. British Medical fournal, 339, b2684.

Kleindl, R. (2010). Slackline-die kunst des modernen seiltanzes. Aachen: Meyer \& Meyer Verlag.

Madigan, M. L., Davidson, B. S., \& Nussbaum, M. A. (2006). Postural sway and joint kinematics during quiet standing are affected by lumbar extensor fatigue. Human Movement Science, 25(6), 788-799.

McGuine, T. A., Greene, J. J., Best, T., \& Leverson, G. (2000). Balance as a predictor of ankle injuries in high school basketball players. Clinical fournal of Sport Medicine, 10(4), 239-244.

McGuine, T. A., \& Keene, J. S. (2006). The effect of a balance training program on the risk of ankle sprains in high school athletes. American Fournal of Sports Medicine, 34(7), 11031111.

Miller, F., \& Friesinger, F. (2009). Slackline. Köngen: Panico Alpinverlag.

Muller, O., Gunther, M., Krauss, I., \& Horstmann, T. (2004). Physical characterization of the therapeutic device Posturomed as a measuring device - presentation of a procedure to characterize balancing ability. Biomedizinische Technik/Biomedical Engineering, 49(3), 56-60.

Myer, G. D., Ford, K. R., McLean, S. G., \& Hewett, T. E. (2006). The effects of plyometric versus dynamic stabilization and balance training on lower extremity biomechanics. American fournal of Sports Medicine, 34(3), 445-455.

Myklebust, G., Engebretsen, L., Braekken, I. H., Skjolberg, A., Olsen, O. E., \& Bahr, R. (2003). Prevention of anterior cruciate ligament injuries in female team handball players: A prospective intervention study over three seasons. Clinical fournal of Sport Medicine, 13(2), 71-78.

Paterno, M. V., Myer, G. D., Ford, K. R., \& Hewett, T. E. (2004). Neuromuscular training improves single-limb stability in young female athletes. Fournal of Orthopaedic and Sports Physical Therapy, 34(6), 305-316.

Pedersen, J., Dye, S. F., Bergenheim, M., \& Djupsjöbacka, M. (2000). Peripheral afferents of the knee: Their effects on central mechanisms regulating muscle stiffness, joint stability, and proprioception and coordination. In S. M. Lephart, \& F. H. Fu (Eds.), Proprioception and neuromuscular control in joint stability (pp. 5-22). Leeds, United Kingdom: Human Kinetics.

Rasool, J., \& George, K. (2007). The impact of single-leg dynamic balance training on dynamic stability. Physical Therapy in Sport, 8(4), 177-184.

Riemann, B. L., Myers, J. B., \& Lephart, S. M. (2003). Comparison of the ankle, knee, hip, and trunk corrective action shown during single-leg stance on firm, foam, and multiaxial surfaces. Archives of Physical Medicine and Rehabilitation, 84(1), 90-95.

Rietdyk, S., Patla, A. E., Winter, D. A., Ishac, M. G., \& Little, C. E. (1999). NACOB presentation CSB New Investigator Award. Balance recovery from medio-lateral perturbations of the upper body during standing. North American Congress on Biomechanics. Fournal of Biomechanics, 32(11), 1149-1158.

Shumway-Cook, A., \& Woollacott, M. H. (2007). Motor control: Translating research into clinical practice. Philadelphia: Williams \& Wilkins.

Taube, W., Gruber, M., Beck, S., Faist, M., Gollhofer, A., \& Schubert, M. (2007). Cortical and spinal adaptations induced by balance training: Correlation between stance stability and corticospinal activation. Acta Physiologica (Oxford, England), 189(4), 347-358.

Taube, W., Leukel, C., \& Gollhofer, A. (2008). Influence of enhanced visual feedback on postural control and spinal reflex 
modulation during stance. Experimental Brain Research, 188(3), 353-361.

Valovich McLeod, T. C. (2008). The effectiveness of balance training programs on reducing the incidence of ankle sprains in adolescent athletes. Fournal of Sport Rehabilitation, 17(3), 316323.

Wang, H. K., Chen, C. H., Shiang, T. Y., Jan, M. H., \& Lin,

K. H. (2006). Risk-factor analysis of high school basketball- player ankle injuries: A prospective controlled cohort study evaluating postural sway, ankle strength, and flexibility. Archives of Physical Medicine and Rehabilitation, 87(6), 821825.

Williams, G. N., Chmielewski, T., Rudolph, K., Buchanan, T. S., \& Snyder-Mackler, L. (2001). Dynamic knee stability: Current theory and implications for clinicians and scientists. fournal of Orthopaedic and Sports Physical Therapy, 31(10), 546-566. 